\title{
Comparison Between Plethysmometer and Caliper Methods to Monitor Lesion-Size Induced by Leishmania major Infection in BALB/c Mouse Experimental Model
}

\author{
Hanene Attia ${ }^{1,2}$, Aymen Bali ${ }^{1,2}$, Rabiaa M. Sghaier ${ }^{1,2}$, Pablo A. Leon Martinez ${ }^{1,2}$, \\ Ghada Mkannez $^{1,2}$, Chiraz Atri ${ }^{1,2}$, Khaled Chourabi ${ }^{1,2}$, Fatma Z. Guerfali ${ }^{1,2}$, Dhafer Laouini ${ }^{1,2, *}$ \\ ${ }^{1}$ Institut Pasteur de Tunis, LR11IPT02, Laboratory of Transmission, Control and Immunobiology of Infections (LTCII), Tunis-Belvédère, \\ Tunisia \\ ${ }^{2}$ Université Tunis El Manar, Tunis, Tunisia
}

Email address:

dhafer_1@yahoo.ca (D. Laouini), dhafer.laouini@pasteur.rns.tn (D. Laouini)

To cite this article:

Hanene Attia, Aymen Bali, Rabiaa M. Sghaier, Pablo A. Leon Martinez, Ghada Mkannez, Chiraz Atri, Khaled Chourabi, Fatma Z. Guerfali, Dhafer Laouini. Comparison Between Plethysmometer and Caliper Methods to Monitor Lesion-Size Induced by Leishmania major Infection in BALB/c Mouse Experimental Model. Animal and Veterinary Sciences. Vol. 3, No. 6, 2015, pp. 179-185. doi: 10.11648/j.avs.20150306.16

\begin{abstract}
In several parasitological diseases, as for leishmaniasis, measurement of the size of cutaneous lesions, which develop at the site of parasite inoculation in animal models, are the most commonly used index to assess disease progression, to compare parasites pathogenicity or virulence and to determine the effects of drug treatment and immunotherapies. The aim of this study is to compare the accuracy of two measurement tools i.e., the caliper and the plethysmometer to refine the lesion size determination. Our findings showed that the use of plethysmometer produced higher correlation with the importance of the lesion mass at experimental endpoints. These findings suggest that, for better differentiation in drug monitoring or Leishmania (L.) strains' virulence and pathogenicity, plethysmometer method is more sensitive to detect parasite-induced swelling and lesions differences at the end of experimental protocols when lesion size is important but caliper is more indicated for small lesions.
\end{abstract}

Keywords: Leishmania major, BALB/c Experimental Model, Lesion Size, Caliper, Plethysmometer

\section{Introduction}

The clinical manifestations of human cutaneous leishmaniasis caused by Leishmania (L.) major display a spectrum of disease severity varying from an asymptomatic infection to multiple disfiguring scars $[1,2]$. This differential clinical expression is considered as the result of interaction between individual behaviour of the microorganism and the host immunologic background. Relative contribution of the host immune background and the vector components involvement are difficult to measure $[1,3,4]$, especially in host-outbred individuals such as humans.

Animal models of cutaneous leishmaniasis are useful tools for dissecting mechanisms related to disease pathogenesis and vaccine or drug development [5]. Indeed, cutaneous infection of mice with L. major is a well-established experimental model of chronic human disease caused by an intracellular parasite. This local infection is not well controlled by the immune anti-parasite responses in susceptible BALB/c mice and the disease disseminates towards the visceral organs with fatal outcome.

Upon experimental infection with different $L$. major strains, distinct aspects of the clinical spectrum of human cutaneous leishmaniasis have been generally reflecting the spectrum of clinical manifestations in humans with reasonable success. Indeed, infection of different inbred strains of mice with L. major can, to certain limits, mimic the disease phenotype observed in human cutaneous leishmaniasis [6] and is routinely used for vaccine or treatment discovery [7-9].

This model is also suitable for comparing the intrinsic virulence of different isolates of Leishmania belonging to the same species [1, 10] and might hence reflect the pathogenicity and virulence of each isolate. It is also suitable for the determination of the effects of drug treatment, immunotherapies and vaccines.

Several previous $[11,12]$ as well as new [13-15] studies 
used routinely the caliper to monitor the lesion development induced in the footpad of animals. This measure represents the difference between the widths and/or the thicknesses of the infected and the uninfected paws of the same mouse. Hence, caliper measurements are commonly collected along the longest two dimensions of the lesion $\mathrm{x} / \mathrm{y}$ plane only, with the zaxis dimension assumed to be the same [16]. Depending on the manipulator, caliper measures may suffer errors and are not easily reproducible from an experiment to another.

Another measure tool is more and more used i. e., the plethysmometer. It is routinely used to study tumors and potential anti-inflammatory or anti-edema properties of pharmacological substances [17-20]. It is more and more routinely used to measure paw inflammation and edema in rodent models [21, 22]. Instead of measuring the lesion diameter, the size is rather determined by the difference between the volume of the infected paw and the volume of the uninfected one of the same mouse. To our knowledge, there are no studies reporting comparison between the two methods in the experimental model of $L$. major infection.

In this work, using the BALB/c experimental mice model, we aimed to compare lesion measurements obtained by both methods on the same animals infected with different $L$. major wild strains isolated from patients living in a zoonotic cutaneous leishmaniasis (ZCL) endemic area of Tunisia.

\section{Materials and Methods}

Eleven strains of L. major were isolated from fresh lesions of patients living in the Centre of Tunisia, an endemic region for ZCL. The study protocol, consent forms and procedures were reviewed and approved by the Institut Pasteur de Tunis Ethical Review Board. Patients provided written informed consent for the collection of parasites and subsequent use.

The isolates were harvested between October 2009 and March 2010, the period in which the lesions of the majority of the individuals infected during the ZCL transmission period appear.

\subsection{Parasite Culture, Metacyclic form Purification}

Promastigotes were cultured in NNN solid medium at $26^{\circ} \mathrm{C}$, and then progressively adapted to a medium composed of RPMI 1640 and $10 \%$ of Fetal Calf Serum (FCS) and supplemented with $100 \mathrm{U}$ of penicillin/ml, $100 \mu \mathrm{g}$ of streptomycin/ml and $2 \mathrm{mM}$ L-glutamine. When the stationary phase was reached, the metacyclic promastigotes were then purified by a negative selection with peanut agglutinin (PNA) (Sigma, Saint-Quentin Fallavier, France). The $\mathrm{PNA}^{-}$and $\mathrm{PNA}^{+}$fractions were separated by density gradient centrifugation. The PNA-metacyclic promastigotes were washed and diluted at the desired density.

\subsection{Animal Infection and Monitoring of the Disease Pattern}

$\mathrm{BALB} / \mathrm{c}$ female mice were obtained from the specific pathogen-free animal-breeding facility Janvier (France) and maintained in a conventional animal facility at Institut Pasteur de Tunis, Tunisia. Animal experiments were performed in compliance with the directive 86/609/EEC of the European parliament and the council on the protection of animals used for scientific purposes, in agreement with the guidelines of International Guiding Principles for Biomedical Research Involving Animals and with the approval of the Ethical Review Board of Institut Pasteur de Tunis.

For each Leishmania isolate (identified by an Arabic number), a group of 5 to 6 eight-week old BALB/c mice was infected with $2 \times 10^{6}$ metacyclic promastigotes. Isolates correspond to parasites obtained from ZCL patients with heterogeneous clinical outcomes. The parasites were suspended in $50 \mu \mathrm{l}$ of $1 \mathrm{X}$ PBS and injected in the left footpad. The right footpad is considered as an uninfected control.

From the second week of infection, lesion development was assessed weekly by measuring the diameter and volume of right and left pads by the same operator and using the same devices i. e.; a vernier caliper and a plethysmometer (Model 7140; Ugo Basile, Italy). Lesion development using caliper was then assessed by measuring the thickness diameter difference between the infected and non-infected footpad of every mouse ( $\mathrm{mm})$. Immediately following measurements, data sets were also collected using plethysmometer device.

The water digital plethysmometer is formed by two interconnected tubes filled with a conductive solution, a platinum electrode for each chamber and a control unit. When the mouse paw is immersed in the measuring tube, the water displacement is also produced in the second tube inducing a change in the conductance between the two platinum electrodes. The control unit detects the conductance changes and generates an output signal to the digital display indicating the volume displacement measured in milliliter (0.01 $\mathrm{ml}$ resolution). Difference between the volume displacement of the infected and uninfected footpad indicates the lesion volume $(\mathrm{ml})$. At the end of the experimental protocol, mice were sacrificed and the infected and contralateral footpads of each mouse were excised and weighed using a precision balance. Parasite load from infected mice was determined by limiting-dilution method following a protocol described elsewhere [23]. Briefly, excised pads were homogenized. A serial 10-fold dilutions were plated in duplicate in 96-well flat-bottom microtiter plates (Nunc, Roskilde, Denmark) containing Schneider's Drosophila medium (both from Gibco-BRL, Paisley, Scotland) supplemented with $100 \mathrm{U}$ of penicillin/ml, $100 \mu \mathrm{g}$ of streptomycin $/ \mathrm{ml}, 2 \mathrm{mM}$ L-glutamine, and $10 \%$ heatinactivated fetal calf serum. Viable parasites were observed microscopically after seven to 10 days of incubation at $26^{\circ} \mathrm{C}$. Parasite load was then determined as the reciprocal of the highest dilution at which promastigotes could be grown in culture at $26^{\circ} \mathrm{C}$ and detected by microscope.

\subsection{Statistical Analyses}

All the statistical tests were performed using PRISM software. To measure the correlation between the lesion size 
obtained either using a plethysmometer or a caliper, the Pearson correlation coefficient $r$ and the $p$ value were used. To evaluate the sensitivity of plethysmometer method versus caliper one, number of variables above or below the linear regression were quantified.

\section{Results}

\subsection{Lesion Size Monitoring}

For each tested Leishmania isolate, a group of five to six $\mathrm{BALB} / \mathrm{c}$ mice were infected. Footpad sizes were weekly measured during eight weeks using both the caliper and the plethysmometer. The sizes of the contralateral footpads of each mouse were also measured using both methods in the same day of the former measurement.

A

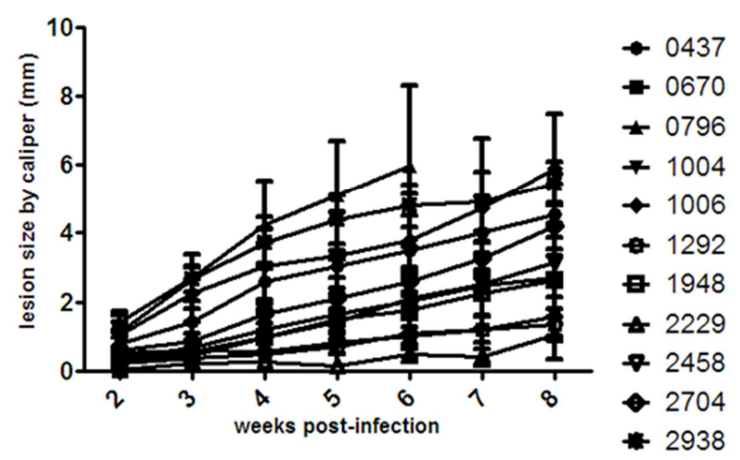

B

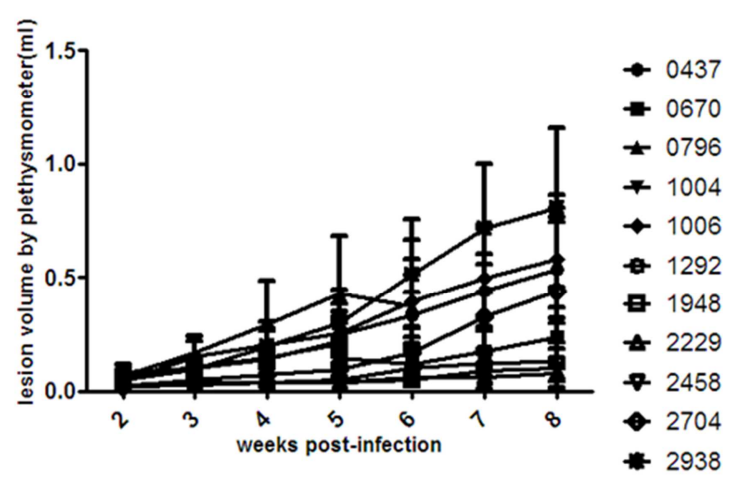

Figure 1. Lesion evolution in BALB/c mice infected with different wild Leishmania major field isolates. Left hind footpad Mice were inoculated subcutaneously with $2 \times 10^{6}$ metacyclic promastigotes. Measurements were performed weekly using a vernier caliper (A) and a plethysmometer (B). Lesion diameter (A) and volume (B) were calculated by subtracting the measure of the contralateral-uninfected footpad using the same tool. Arabic numbers corresponding to each isolate mentions the parasite identification. Results are expressed as the mean lesion size of five to six mice per group \pm $S D$.

As shown in Fig. 1, lesion evolution in each group of mice was measured either with caliper (Panel A) or plethysmometer (Panel B). Results showed a heterogeneous evolution of lesions during the infection course, according to the parasite isolate used. When comparing the two methods for each isolate, we did not notice any important difference according to the lesion evolution. Nevertheless, scale extent seems to be more pronounced at the beginning or the end of infection course, when measures were taken by caliper or by plethysmometer, respectively.

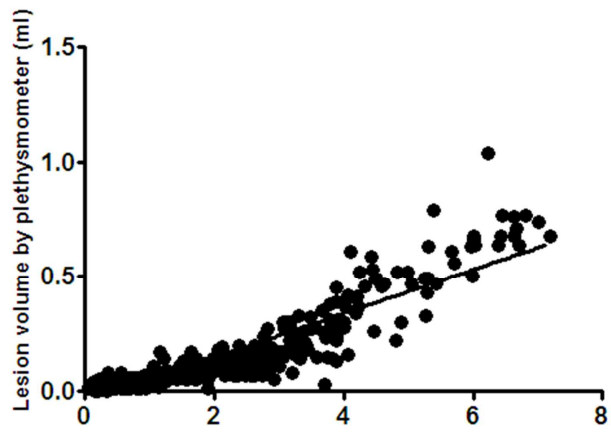

Lesion size by caliper $(\mathrm{mm})$

Figure 2. Pair-comparison and linear regression of 362 plethysmometer measurements against their caliper counterparts of lesion sizes during eightweek protocol. Caliper measurements were expressed by $\mathrm{mm}$ and plethysmometer measurements were expressed by $\mathrm{ml}$ and correspond respectively to the diameter and the volume of each lesion as the difference between the infected and uninfected footpad. Pearson $r$ correlation coefficients were 0.9070 and 0.8227 respectively.

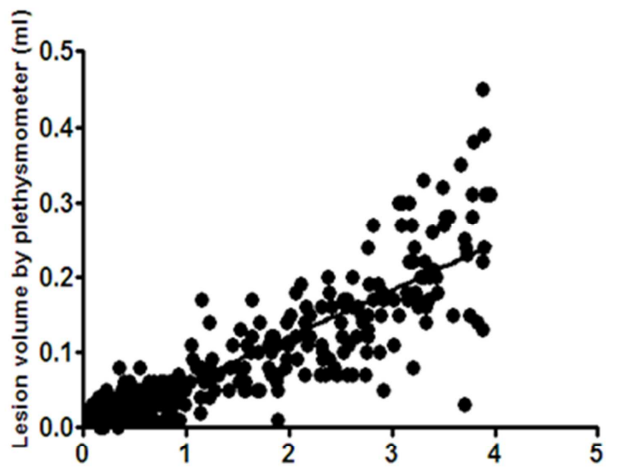

Lesion size by caliper $(\mathrm{mm})$

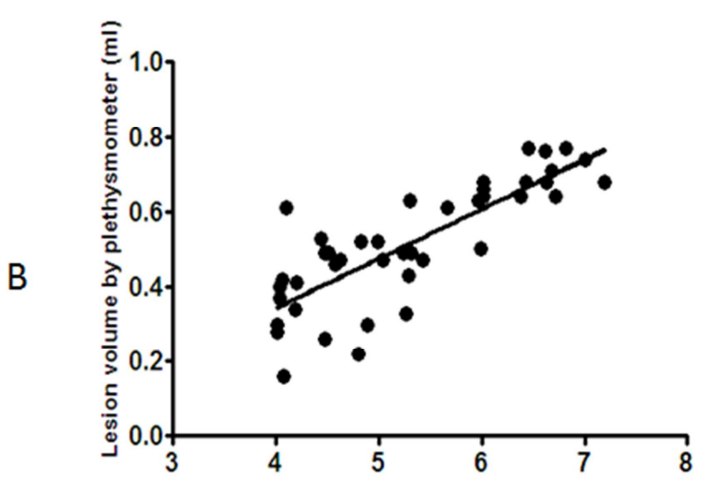

Lesion size by caliper $(\mathrm{mm})$

Figure 3. Pair-comparison and linear regression of caliper measurements against their plethysmometer counterparts of lesion sizes depending on their diameter range. Panel A corresponds to lesion diameter $<4 \mathrm{~mm}(r=0.8578)$ and panel $B$ corresponds to lesion diameter $>4 \mathrm{~mm}(r=0.8089)$. Caliper measurements were expressed by $\mathrm{mm}$ and plethysmometer measurements were expressed by $m l$ and correspond respectively to the diameter and the volume of each lesion after calculating the difference between the infected and uninfected footpads. 


\subsection{Correlation, Sensitivity and Reproducibility Between Plethysmometer and Caliper}

Lesion size obtained with plethysmometer and caliper were then pair-compared for each mouse at each time point. The distribution of the total 362 paired-measures was plotted. Results (Fig. 2) showed that global correlation between these paired-measures is statistically significant with a Pearson $r$ coefficient significantly close to 1 (Pearson $r=0.9070$ and $p<$ $0.0001)$. This result witnesses a high correlation between the measures generated using the caliper and those using the plethysmometer.

To assess the sensitivity of each method towards the other, we considered number of points above and below the linear regression curve.

Points above the regression line were more numerous than those below the line (200 versus 162 , respectively) indicating that sensitivity is more important by plethysmometer than caliper.

Interestingly, paired-measures' points above the regression line are more numerous when lesion measurements are higher (by both methods) and vice versa. Accordingly, we separated the paired-measures lower or higher than $4 \mathrm{~mm}$ as measured by caliper and compared points of each group (range 1 and range 2 respectively, Fig. 3). When comparing

A

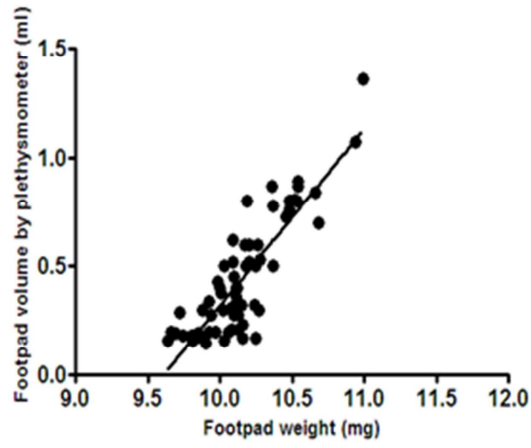

C

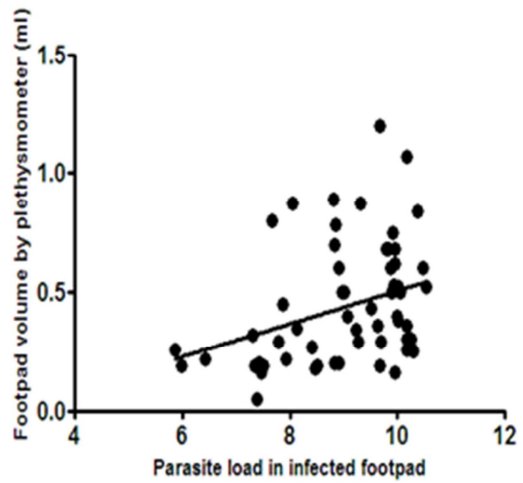

paired-measures at range 1 , (lower than $4 \mathrm{~mm}$ by caliper), Pearson $r=0.8578$ and $p<0.0001$ and when comparing measures at range 2 (higher than $4 \mathrm{~mm}$ by caliper), Pearson $r=0.8089$ and $p<0.0001$. Points above the line are more numerous at range 2 (24 out of $42 ; 57 \%$; Fig. 3, Panel B) than at range 1 ( 155 out of 313 ; 49\%; Fig. 3, Panel A).

All these results indicate that sensitivity of plethysmometer measurements seems higher than caliper ones with bigger lesions than with smaller ones and vice versa.

To assess reproducibility, we assumed that diameter and volume of each non-infected footpad would be the same during the whole time of experiment. Hence, measures of diameter and volume of contralateral non-infected pads were weakly taken by the same experimenter using both plethysmometer and caliper. Intra-observational variations were $2,5 \%(2.45 \pm 0,06 \mathrm{~mm}$; mean $\pm \mathrm{SD})$ in caliper measures and $15,1 \%(0.13 \pm 0.02 \mathrm{ml}$; mean $\pm \mathrm{SD})$ in plethysmometer measures. This result indicates that measures using caliper were more reproducible than those taken with plethysmometer. However, this should be taken with caution since all measures of non-infected footpad are within a range where caliper is more sensitive.

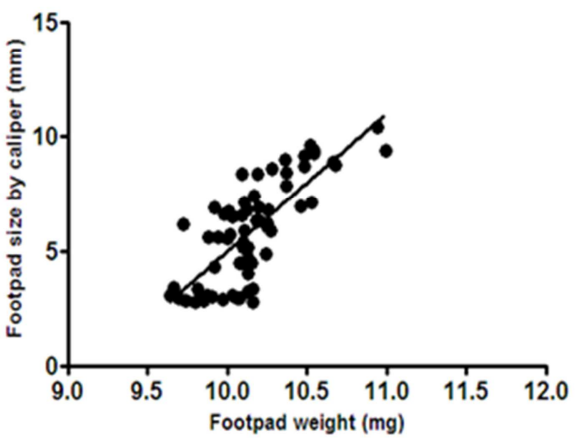

D

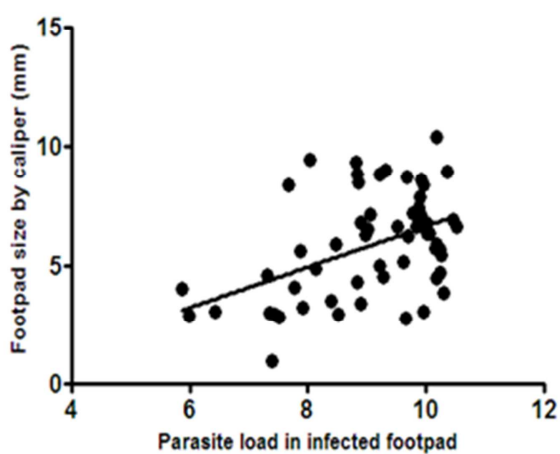

Figure 4. Pair-comparison and linear regression of plethysmometer and caliper measurement of infected footpads size against footpad weight (panels A and $B$ ) and parasite load (panels $C$ and D). Panel A corresponds to plethysmometer measurement versus footpad weight ( $r=0.8515$ ), panel B to caliper measurement versus footpad weight $(r=0.7936)$, panel $C$ to plethysmometer measurement versus parasite load ( $r=0.3239)$ and panel $D$ to caliper measurement versus parasite load $(r=0.4634)$. 


\subsection{Correlation Between Plethysmometer or Caliper Measures and Footpad Weight or Parasite Load}

At the end of the eight weeks, mice were sacrificed and the infected feet were excised and weighed. Parasite load was also monitored. The foot weight and the parasite load were used as golden standard measures to evaluate the accuracy of each method. Accuracy can be defined as proximity between the measure of an object and its true value. Indeed, the foot weight is likely reflecting the direct effect of a given isolate on tissue inflammation and lesional extent and the parasite load might be correlated with the apparent lesion size.

As shown in Fig. 4, the volume of the whole infected footpad determined by plethysmometer correlated with footpad weight (Pearson $r=0.8515$; Fig. 4 , panel A) slightly better than the diameter of the whole infected footpad measured with caliper (Pearson $r=0.7936$; Fig. 4, panel B). When splitting the measurements into two ranges, in those higher than $4 \mathrm{~mm}$ by caliper (data not shown), correlations still slightly higher between plethysmometer measurements and footpad weight than those between caliper measurements and footpad weight (Pearson $r=0.9436$ and 0.8852 respectively). In $<4 \mathrm{~mm}$ diameters, correlations between plethysmometer measurements and footpad weight are however faintly lower than those between caliper measurements and footpad weight ( $r=0.8835$ and 0.9435 respectively).

When comparing the diameter and volume measures with footpad parasite load, Pearson coefficients are much lower with a slight better correlation with caliper measurements (Pearson $r=0.4634$; Fig. 4, panel D) then with plethysmometer measurements (Pearson $r=0.3239$; Fig. 4, panel C). These very low correlations indicate that parasite load cannot be considered, like footpad weight, as a good standard for lesion monitoring.

In conclusion, all these results indicate that measures undertaken either by plethysmometre or caliper are comparable and not revealing notable differences. Nevertheless, when lesions are important, the measures made by plethysmometre are dimly better correlated with infected footpad weight than those undertaken using caliper. However, caliper is better to be used when lesions are small.

\section{Discussion}

In order to compare two measurement methods for lesion development, Tunisian isolates of L. major freshly collected from the field were tested for their experimental pathogenicity in BALB/C mice model. Lesion development was assessed during the infection course using both the caliper and the plethysmometer. Despite the strong correlation between the two methods, power discrimination between the isolates when measures were taken by caliper or plethysmometer was different depending on the lesion size. Indeed, for lesion diameter lower than $4 \mathrm{~mm}$ by caliper, plethysmometer is less sensitive but when lesion size is higher than $4 \mathrm{~mm}$, caliper is less sensitive.
Interestingly, when correlating the lesions diameters or volumes obtained by both methods, with total infected foot weight as a standard for lesion development, plethysmometer shows faintly more accurate measures; whereas caliper method seems more accurate when correlating its measures with the total parasite load in infected pads.

These results indicate that both methods could be used, depending on the objectives.

Many studies argued in favor of the use of plethysmometer instead of caliper. As for parasitological studies, cancer studies for instance, require a precise tool to measure tumor volume. Several authors concluded that caliper is inaccurate and encumbered with a significant and size dependent bias compared to other methods [24, 25]. Indeed, and despite its low cost, caliper method was often affected by much error, subjective and inaccurate especially for large tumors which shape differs from small ones [24]. To measure pathogeninduced edema and hyperalgesia during inflammation, other authors used the plethysmometer rather than the caliper for its accuracy [26]. Other physical methods have been shown superior to caliper measurements for determining xenograft tumor volume in mouse models [27].

However, and as far as we know, only one study suggested that the micrometer method, a method comparable to caliper one, could be more sensitive and useful in measuring paw edema in mice than the plethysmometer method, when used to assess the anti-inflammatory activity [28]. Besides the mice models, a recent study presented a precise, complete and noncontact 3D assessment tool for monitoring the evolution of human cutaneous leishmaniasis wounds based on a 3D laser scanner and computer vision algorithms [29]. This proposed 3D assessment tool showed higher accuracy and precision rates than conventional manual methods.

We conclude that use of plethysmometer to measure lesion volume in Leishmania mouse model will result in more sensitive measures of volume change at endpoints, especially when lesions are important, like those induced by high virulent strains. However, the caliper method, the current gold standard for lesion measurement in experimental leishmaniasis shows a slight more reproducibility and should be used to monitor lesion size for anti-Leishmania vaccines or drugs, where the lesions are expected to be smaller.

\section{Conclusion}

In conclusion, the use of caliper or plethysmometer for monitoring disease progression in Leishmania infected mice should depend mainly on the study goals, having in mind the limitations of each tool and used methodology towards better accuracy. Hence, plethysmometer method showed more sensitivity at the end of experimental protocols when lesion size is important but caliper is more indicated for small lesions.

\section{Acknowledgments}

We thank Mr Ammar Marouani and Dr Zakaria Ben 
Lasfar, Animal Facilities of the Institut Pasteur de Tunis for their help conducting this study. This work was supported by an NIH/NIAID/DMID Grant Number 5P50AI074178 for and Tunisian Ministry of Higher Education and Research for DL. The funders had no role in study design, data collection and analysis, decision to publish, or preparation of the manuscript.

\section{References}

[1] Kebaier C, Louzir H, Chenik M, Ben Salah A, Dellagi K. Heterogeneity of wild Leishmania major isolates in experimental murine pathogenicity and specific immune response. Infect Immun. 2001; 69 (8): 4906-15.

[2] Van Thiel PP, van Gool T, Faber WR, Leenstra T, Kager PA, Bart A. Variation in clinical presentation and genotype of causative Leishmania major strain in cutaneous leishmaniasis in north and south Afghanistan. Am J Trop Med Hyg. 2011; 85 (1): 60-3.

[3] Lima HC, Titus RG. Effects of sand fly vector saliva on development of cutaneous lesions and the immune response to Leishmania braziliensis in BALB/c mice. Infect Immun. 1996; 64 (12): 5442-5.

[4] Rohousova I, Volf P. Sand fly saliva: effects on host immune response and Leishmania transmission. Folia Parasitol (Praha). 2006; 53 (3): 161-71.

[5] Baldwin TM, Elso C, Curtis J, Buckingham L, Handman E. The site of Leishmania major infection determines disease severity and immune responses. Infect Immun. 2003; 71 (12): 6830-4.

[6] Sacks D, Noben-Trauth N. The immunology of susceptibility and resistance to Leishmania major in mice. Nat Rev Immunol. 2002; 2 (11): 845-58.

[7] Soto M, Corvo L, Garde E, Ramirez L, Iniesta V, Bonay P et al. Coadministration of the Three Antigenic Leishmania infantum Poly (A) Binding Proteins as a DNA Vaccine Induces Protection against Leishmania major Infection in BALB/c Mice. PLoS Negl Trop Dis. 2015; 9 (5): e0003751.

[8] Fortin A, Caridha DP, Leed S, Ngundam F, Sena J, Bosschaerts $\mathrm{T}$ et al. Direct comparison of the efficacy and safety of oral treatments with oleylphosphocholine (OIPC) and miltefosine in a mouse model of L. major cutaneous leishmaniasis. PLoS Negl Trop Dis. 2014; 8 (9): e3144.

[9] Martinez Salazar MB, Delgado Dominguez J, Silva Estrada J, Gonzalez Bonilla C, Becker I. Vaccination with Leishmania mexicana LPG induces PD-1 in CD8(+) and PD-L2 in macrophages thereby suppressing the immune response: a model to assess vaccine efficacy. Vaccine. 2014; 32 (11): 1259-65.

[10] Meddeb-Garnaoui A, Zrelli H, Dellagi K. Effects of tropism and virulence of Leishmania parasites on cytokine production by infected human monocytes. Clin Exp Immunol. 2009; 155 (2): 199-206.

[11] Soong L, Duboise SM, Kima P, McMahon-Pratt D. Leishmania pifanoi amastigote antigens protect mice against cutaneous leishmaniasis. Infect Immun. 1995; 63 (9): 355966.
[12] Brown DR, Swier K, Moskowitz NH, Naujokas MF, Locksley RM, Reiner SL. T helper subset differentiation in the absence of invariant chain. J Exp Med. 1997; 185 (1): 31-41.

[13] Cortez M, Huynh C, Fernandes MC, Kennedy KA, Aderem A, Andrews NW. Leishmania promotes its own virulence by inducing expression of the host immune inhibitory ligand CD200. Cell Host Microbe. 2011; 9 (6): 463-71.

[14] Xu W, Xin L, Soong L, Zhang K. Sphingolipid degradation by Leishmania major is required for its resistance to acidic $\mathrm{pH}$ in the mammalian host. Infect Immun. 2011; 79 (8): 3377-87.

[15] Spath GF, Garraway LA, Turco SJ, Beverley SM. The role(s) of lipophosphoglycan (LPG) in the establishment of Leishmania major infections in mammalian hosts. Proc Natl Acad Sci U S A. 2003; 100 (16): 9536-41.

[16] Tomayko MM, Reynolds CP. Determination of subcutaneous tumor size in athymic (nude) mice. Cancer Chemother Pharmacol. 1989; 24 (3): 148-54.

[17] Uzcategui B, Avila D, Suarez-Roca H, Quintero L, Ortega J, Gonzalez B. Anti-inflammatory, antinociceptive, and antipyretic effects of Lantana trifolia Linnaeus in experimental animals. Invest Clin. 2004; 45 (4): 317-22.

[18] Romero-Sandoval EA, Molina C, Alique M, MorenoManzano V, Lucio FJ, Herrero JF. Vitamin A active metabolite, all-trans retinoic acid, induces spinal cord sensitization. I. Effects after oral administration. $\mathrm{Br} \mathrm{J}$ Pharmacol. 2006; 149 (1): 56-64.

[19] Ratnasooriya WD, Deraniyagala SA, Galhena G, Liyanage SSP, Bathige SDNK, Jayakody RAC. Anti-inflammatory Activity of the Aqueous Leaf Extract of Ixora coccinea. Pharmaceutical Biology. 2005; 43 (2): 147-52.

[20] Raut MK, Bhandari SV, Bothara KG, Patil AA, Sarkate AP. Anti-inflammatory, analgesic, ulcerogenic, activities of some new non-acidic diclofenac and 1, 3, 4-oxadiazole derivatives in aanimal models Pharmacologyonline. 2007; 2: 172-86.

[21] Vazquez E, Navarro M, Salazar Y, Crespo G, Bruges G, Osorio $\mathrm{C}$ et al. Systemic changes following carrageenan-induced paw inflammation in rats. Inflamm Res. 2015; 64 (5): 333-42.

[22] De Toni LG, Menaldo DL, Cintra AC, Figueiredo MJ, de Souza AR, Maximiano WM et al. Inflammatory mediators involved in the paw edema and hyperalgesia induced by Batroxase, a metalloproteinase isolated from Bothrops atrox snake venom. Int Immunopharmacol. 2015; 28 (1): 199-207.

[23] Benhnini F, Chenik M, Laouini D, Louzir H, Cazenave PA, Dellagi K. Comparative evaluation of two vaccine candidates against experimental leishmaniasis due to Leishmania major infection in four inbred mouse strains. Clin Vaccine Immunol. 2009; 16 (11): 1529-37.

[24] Jensen MM, Jorgensen JT, Binderup T, Kjaer A. Tumor volume in subcutaneous mouse xenografts measured by microCT is more accurate and reproducible than determined by $18 \mathrm{~F}-F D G-m i c r o P E T$ or external caliper. BMC Med Imaging. 2008; 8: 16.

[25] Girit IC, Jure-Kunkel M, McIntyre KW. A structured lightbased system for scanning subcutaneous tumors in laboratory animals. Comp Med. 2008; 58 (3): 264-70.

[26] Chanana V, Sehgal R, Rishi P. Salmonella typhi iron-regulated outer-membrane proteins cause oedema and hyperalgesia during inflammation induced in a rat model. J Med Microbiol. 2005; 54 (Pt 4): 421-3. 
[27] Fiennes AG. Growth rate of human tumour xenografts measured in nude mice by in vivo cast modelling. Br J Surg. 1988; 75 (1): 23-4.

[28] Sharma JN, Samud AM, Asmawi MZ. Comparison between plethysmometer and micrometer methods to measure acute paw oedema for screening anti-inflammatory activity in mice. Inflammopharmacology. 2004; 12 (1): 89-94.
[29] Zvietcovich F, Castaneda B, Valencia B, Llanos-Cuentas A. A $3 \mathrm{D}$ assessment tool for accurate volume measurement for monitoring the evolution of cutaneous leishmaniasis wounds. Conf Proc IEEE Eng Med Biol Soc. 2012; 2012: 2025-8. 